\title{
Parentage analysis of tea cultivars in Japan based on simple sequence repeat markers
}

\author{
Nakao Kubo*1,2), Tomohiro Matsuda ${ }^{3)}$, Chisaki Yanagida', Yuna Hotta', Yutaka Mimura ${ }^{\text {() }}$ and Maho Kanda ${ }^{3)}$ \\ 1) Graduate School of Life and Environmental Sciences, Kyoto Prefectural University, 1-5 Hangi-cho, Shimogamo, Sakyo-ku, Kyoto \\ 606-8522, Japan \\ 2) Biotechnology Research Department, Kyoto Prefectural Agriculture, Forestry and Fisheries Technology Center, 74 Oji, \\ Kitainayazuma, Seika-cho, Soraku-gun, Kyoto 619-0244, Japan \\ 3) Tea Industry Research Division, Agriculture and Forestry Technology Department, Kyoto Prefectural Agriculture, Forestry and \\ Fisheries Technology Center, 1 Nakanosono, Shirakawa, Uji, Kyoto 611-0022, Japan
}

Tea cultivars have been bred by individual selection of landraces and by crossbreeding, but the validation of the parentage is limited. In this study, we performed parentage analysis of 79 tea cultivars in Japan based on SSR markers to confirm or identify the parent-offspring relationships among them. The effectiveness of nine SSR markers for parentage analysis was validated by comparing them to the existing cleaved amplified polymorphic sequence markers. The former markers were detectable more alleles than the latter. Simulation of parentage analysis of the tea cultivars predicted biparental origins for 12 cultivars ('Houshun', 'Mie ryokuhou no. 1', 'Surugawase', 'Tenmyo', 'Yamanoibuki', 'Harumidori', 'Koushun', 'Minekaori', 'Okumusashi', 'Saemidori', 'Sofu', and 'Toyoka'), in the first five of which candidate parents of yet-to-bedefined pedigree were newly identified. Comparisons of a total of 41 SSR genotypes confirmed the newlyidentified parentages of 'Asahi' for 'Tenmyo', 'Rokurou' for 'Houshun', 'Surugawase', and 'Yamanoibuki', and 'Yamatomidori' for 'Mie ryokuhou no. 1'. The maternity of seven cultivars out of the 12 was also confirmed with chloroplast DNA sequences. Uniparental origins were confirmed for 25 cultivars. This parentage analysis has improved our knowledge of tea pedigrees and will aid in the development of new cultivars.

Key Words: DNA marker, parentage analysis, pedigree, simple sequence repeat (SSR), tea (Camellia sinensis) cultivar.

\section{Introduction}

Tea (Camellia sinensis (L.) Kuntze) is a species of evergreen tree that is important for making beverages. This species includes two major varieties, $C$. sinensis var. sinensis (L.) Kuntze and $C$. sinensis var. assamica (J. W. Mast.) Kitam. (see Chen and Chen 2012 for a review). Its geographic origin is predicted to be southwestern China, and teas are now cultivated in various tropical, sub-tropical, and temperate regions worldwide. Because tea plants undergo outcrossing due to self-incompatibility, seed progenies generally have different allele combinations from parents as well as among siblings. Long-established tea gardens used to be composed of heterogenic, seed-derived populations, known as landraces; in the modern era, individuals with superior traits have been selected and vegeta-

Communicated by Masafumi Yagi

Received December 4, 2020. Accepted July 9, 2021.

First Published Online in J-STAGE on October 14, 2021.

*Corresponding author (e-mail: nkubo@kpu.ac.jp) tively propagated as cultivars. To date, hundreds of tea cultivars have been bred in leading tea-producing countries, such as China, India, Sri Lanka, Kenya, and Japan (Chen and Chen 2012). In Japan, tea is an important beverage in tea ceremonies (Cha-no-yu or Sado) and in daily life. Japanese tea has recently attracted foreign consumers and purchasers. Since the introduction of tea to Japan in the medieval era, tea cultivation has expanded from Kyoto throughout most of Japan. The cultivar 'Yabukita', which is derived from a Shizuoka landrace, is currently the most widely cultivated tea because of its relatively high yield and wide regional adaptability. Many green tea cultivars have been bred by crossbreeding this cultivar and its relatives (Tanaka 2012).

Green tea processed in Kyoto Prefecture is called " $U j i$ cha" and is known as one of the highest-quality teas in the world. It includes tencha (tea leaves are steamed and dried without rolling, then ground in a stone mill to a fine powder known as matcha) and gyokuro (steamed and rolled green tea made from shade-grown leaves) in addition to sencha, a popular steamed and rolled green tea. Several cultivars 
have been bred by individual selection of Kyoto landraces or their progenies (e.g. 'Asahi', 'Goko', 'Samidori', 'Ujihikari', and 'Ujimidori'). 'Houshun' and 'Tenmyo' are green tea cultivars suitable for making gyokuro and tencha, both of which were selected from naturally pollinated seed progenies of 'Samidori' (Kyoto Prefectural Tea Research Institute 2004a, 2004b). However, their paternal parents are unknown.

Because of the highly outcrossed nature, information on tea pedigrees would be helpful to avoid inbreeding depression in breeding programs. To date, identification and parentage analyses of tea cultivars have been conducted at the DNA level (see Ni et al. 2012 for a review). Such analyses have also been performed in Chinese and Japanese cultivars based on flower morphology (Takeda and Toyao 1980), disease resistance (Takeda 2003), and DNA markers (Katoh et al. 2011, Kaundun and Matsumoto 2003, 2004, Matsumoto 2006, Matsumoto et al. 1994, Tanaka and Yamaguchi 1996, Tanaka et al. 2001, Ujihara et al. 2009, 2011, Zhang et al. 2020). Analyses of DNA markers have shown that parentages were in some cases misunderstood, and the pedigrees of some cultivars have been revised in light of this new information (Matsumoto 2006, Tanaka and Yamaguchi 1996). Among DNA markers, simple sequence repeats (SSRs), which are repeat sequences composed of 1-6 nucleotide motifs, are frequently used as they present several advantages over other DNA markers (Merritt et al. 2015). More recently, next-generation sequencing (NGS)based approaches have also been applied (e.g. Zhang et al. 2020), though there could be limitations of such approaches related to highly repetitive and heterozygous tea genomes (Xia et al. 2020). Actually, we initially tried an analysis using single nucleotide polymorphism data obtained from our previous NGS-based approach in 44 cultivars (Kubo et al. 2019), but failed in accurate parentage prediction (data not shown) due to the above limitations. Classification of tea landraces and cultivars has been performed based on SSR markers (e.g. Kubo et al. 2019, Liu et al. 2017, Meegahakumbura et al. 2016, 2018, Ohsako et al. 2008, Tamaki et al. 2016, Taniguchi et al. 2014, Wambulwa et al. 2016, 2017). These analyses have revealed relationships among tea varieties, but the pedigrees of these cultivars are yet to be examined. To date, only a limited number of pedigrees have been validated. 'Meiryoku' seems to be derived from a cross between 'Yabukita' and ' $\mathrm{Z1}$ ', whereas 'Yutakamidori' is more likely to be derived from an outcrossing of 'Asatsuyu' instead of selfing (Matsumoto 2006, Tanaka and Yamaguchi 1996). The paternal parental line of 'Okumidori' does not seem to be 'Shizu zai 16' (Matsumoto 2006). Twenty-nine and four cultivars are suggested to be offspring of 'Dabaicha' and 'Tieguanyin', respectively. The parentages of 'Echa 5', 'Foxiang 1', 'Foxiang 2', and 'Huangguanyin' have also been confirmed (Tan et al. 2015), and eight individuals were identified as selfing from 'Ziyan' (Tan et al. 2019).

In the present study, we conducted parentage analysis of selected tea cultivars in Japan, including 'Houshun' and 'Tenmyo', based on SSR markers in order to confirm or newly identify parent-offspring relationships.

\section{Materials and Methods}

\section{Plant materials and DNA extraction}

A total of 79 cultivars, representative of those grown in Japan, were used for analysis (Table 1). For easy identification, each cultivar name is indicated with the sample number in square brackets (e.g. 'AN5' [1]) hereafter. Of these, 36 cultivars were newly included for genotyping in this study (Table 1, asterisks). Fresh leaves of four cultivars ('Kurasawa' [21], 'Sofu' [50], 'Toyoka' [56], and 'Yamatomidori' [66]) were kindly supplied by Tea Industry Research Center, Shizuoka Prefectural Research Institute of Agriculture and Forestry (Kikugawa, Japan), Division of Tea Research, Institute of Fruit Tree and Tea Science, National Agriculture and Food Research Organization (NARO) (Shimada, Japan), Saitama Tea Research Institute (Iruma, Japan), and Yamato Tea Research Center, Nara Prefecture Agricultural Research and Development Center (Nara, Japan), respectively. Fresh leaves of the remaining 32 cultivars were collected from plants grown under natural conditions in tea genetic resource gardens at the Tea Industry Research Division, Agriculture and Forestry Technology Department, Kyoto Prefectural Agriculture, Forestry and Fisheries Technology Center (Uji, Japan), and the University Farm, Faculty of Life and Environmental Sciences, Kyoto Prefectural University (Soraku-gun, Japan).

DNA was extracted with a DNeasy Plant Mini Kit (Qiagen, Valencia, USA) or crude DNA lysate was prepared from small pieces of leaves according to the manufacturer's instructions (KOD FX Neo, Toyobo, Osaka, Japan).

\section{Genotyping of DNA markers}

For validation of the DNA markers in the parentage analysis, three tea cultivars with known pedigrees ('Harumidori' [10], 'Minekaori' [29], and 'Saemidori' [44]; Takeda et al. 1991, 2002, Ueno and Furuno 1989) were examined based on eight cleaved amplified polymorphic sequence (CAPS) markers (Supplemental Table 1; Ujihara et al. 2011). The allele data of the CAPS markers were confirmed to be identical to those in the previous reports (Kitajima-Ujihara 2013, Ujihara et al. 2011), whereas those of 'Unkai' [60] were newly analyzed in this study. Genotypes of the three cultivars were also analyzed based on nine SSR markers (MSG0403, MSG0421, MSG0572, MSG0609, MSG0699, MSG0703, MSG0795, MSG0800, and MSG0811; Taniguchi et al. 2012) (Supplemental Table 1) using fluorescent-labeled primers (Sigma-Aldrich, St. Louis, USA), localized to independent linkage groups (Supplemental Table 3).

The genotypes of 79 cultivars were used to examine the pedigrees based on the nine SSR markers. Genotype data for 43 cultivars were retrieved from our previous study 
Table 1. List of 79 tea cultivars examined in this study

\begin{tabular}{|c|c|c|c|c|c|c|c|c|c|}
\hline Type & No. ${ }^{a}$ & Cultivar $^{b}$ & Pedigree $^{c}$ & & Type & No. ${ }^{a}$ & Cultivar $^{b}$ & Pedigree $^{c}$ & \\
\hline \multicolumn{3}{|c|}{ Green tea cultivar } & & & & 41 & Okuyutaka & Yutakamidori $\times \mathrm{F}_{1} \mathrm{NN} 8$ & \\
\hline & 1 & AN5* & $\mathrm{A} 2 \times \mathrm{S} 24$ & & & 42 & Rokurou* & (Selection of a landrace of unknown origin) & \\
\hline & 2 & Asagiri* & (Selection of Uji landrace) & & & 43 & Ryofu & Houryoku $\times$ Yabukita & \\
\hline & 3 & Asahi & (Selection of Uji landrace) & & & 44 & Saemidori & Yabukita $\times$ Asatsuyu & 卖 \\
\hline & 4 & Asanoka & Yabukita $\times \mathrm{Cp} 1$ & & & 45 & Sakimidori & $\mathrm{F}_{1} \mathrm{NN} 27 \times \mathrm{ME} 52$ & \\
\hline & 5 & Asatsuyu & (Selection of Uji landrace) & & & 46 & Samidori & (Selection of Uji landrace) & \\
\hline & 6 & Fujimidori* & & & & 47 & Sayamakaori & Yabukita $\times$ ? & \\
\hline & 7 & Fukumidori* & Yabukita $\times 23 \mathrm{~F}_{1}-107$ & & & 48 & Sayamamidori* & (Selection of Uji landrace) & \\
\hline & 8 & Fushun & $\mathrm{Z} 1 \times$ Kanayamidori & & & 49 & Shunmei & Yutakamidori $\times \mathrm{F}_{1} \mathrm{NN} 8$ & \\
\hline & 9 & Goko & (Selection of Uji landrace) & & & 50 & Sofu* & Yabukita $\times$ Shizu-inzatsu 131 & 光 \\
\hline & 10 & Harumidori* & Kanayamidori $\times$ Yabukita & 为 & & 51 & Surugawase* & Yabukita $\times ?[$ Rokurou $]$ & $\star$ \\
\hline & 11 & Harumoegi* & $\mathrm{F}_{1} \mathrm{NN} 27 \times \mathrm{ME} 52$ & & & 52 & Takachiho & (Selection of Miyazaki landrace) & \\
\hline & 12 & Hatsumidori* & (Selection of Mie landrace), triploid & & & 53 & Tamamidori* & (Selection of Uji landrace) & \\
\hline & 13 & Himemidori* & (Selection of Fukuoka landrace) & & & 54 & Tenmyo & Samidori $\times ?$ [Asahi] & $\star$ \\
\hline & 14 & Hokumei & Sayamamidori $\times 5507$ & & & 55 & Terakawawase & (Selection of Uji landrace) & \\
\hline & 15 & Hoshinomidori* & (Selection of Fukuoka landrace) & & & 56 & Toyoka* & Sayamamidori $\times$ Yabukita & 支 \\
\hline & 16 & Houshun & Samidori $\times ?$ [Rokurou] & $\star$ & & 57 & Tsuyuhikari* & Shizu $7132 \times$ Asatsuyu & \\
\hline & 17 & Izumi* & Benihomare $\times$ ? & & & 58 & Ujihikari & (Selection of Uji landrace) & \\
\hline & 18 & Kanayamidori & S6 $\times$ Yabukita & & & 59 & Ujimidori & (Selection of Uji landrace) & \\
\hline & 19 & Komakage & (Selection of Uji landrace) & & & 60 & Unkai & Takachiho $\times \mathrm{F}_{1} 9-4-48$ & \\
\hline & 20 & Koushun & Kurasawa $\times$ Kanayamidori & th & & 61 & Yabukita & (Selection of Shizuoka landrace) & \\
\hline & 21 & Kurasawa* & Yabukita $\times$ ? & & & 62 & Yaeho & (Selection of Shizuoka landrace) & \\
\hline & 22 & Kuritawase & (Selection of Shizuoka landrace) & & & 63 & Yamakai & Yabukita $\times$ ? & \\
\hline & 23 & Kyomidori* & (Selection of Uji landrace) & & & 64 & Yamanami* & Seedling introduced from Hubei Province, China & \\
\hline & 24 & Makinoharawase* & (Selection of Shizuoka landrace), triploid & & & 65 & Yamanoibuki* & Yabukita $\times ?[$ Rokurou] & $\star$ \\
\hline & 25 & Meiryoku & Yabukita $\times \mathrm{Z1}^{d}$ & & & 66 & Yamatomidori* & $?^{d}$ & \\
\hline & 26 & Mie ryokuhou no. $1^{*}$ & Yabukita $\times$ ? [Yamatomidori] & $\star$ & & 67 & Yumekaori & Sayamakaori $\times$ Miyazaki no. 8 & \\
\hline & 27 & Minamikaori & Yabukita $\times$ Miya A11 & & & 68 & Yumewakaba* & Yabukita $\times$ Saitama no. 9 & \\
\hline & 28 & Minamisayaka & MiyA- $6 \times \mathrm{F}_{1} \mathrm{NN} 27$ & & & 69 & Yutakamidori* & Asatsuyu $\times ?^{d}$ & \\
\hline & 29 & Minekaori & Yabukita $\times$ Unkai & 幽 & & & & & \\
\hline & 30 & Miyamakaori* & Kyoken $283 \times$ Saitama no. 1 & & Black & and oc & olong tea cultivar & & \\
\hline & 31 & Miyoshi* & (Selection of Uji landrace) & & & 70 & Becchan (Betjan) & & \\
\hline & 32 & Musashikaori & Yabukita $\times 27 \mathrm{~F}_{1}-73$ & & & 71 & Benifuji* & Benihomare $\times \mathrm{C} 19$ & \\
\hline & 33 & Narino & (Selection of Uji landrace) & & & 72 & Benifuuki & Benihomare $\times$ Mak Cd 86 & \\
\hline & 34 & Natsumidori* & (Selection of Shizuoka landrace) & & & 73 & Benihikari* & Benikaori $\times \mathrm{Cn} 1$ & \\
\hline & 35 & NN38* & Yabukita $\times$ Shizu zai 16 & & & 74 & Benihomare & (Selection of Indian hybrids, Tada series) & \\
\hline & 36 & Oguramidori* & (Selection of Uji landrace) & & & 75 & Hatsumomiji* & $\mathrm{Ai} 2 \times \mathrm{NKa05}$ & \\
\hline & 37 & Okuhikari* & Yabukita $\times$ Shizuoka Cy225 & & & 76 & Indo & (Selection of Indian hybrids, Tada series) & \\
\hline & 38 & Okumidori & Yabukita $\times ?^{d}$ & & & 77 & Ooba oolong & & \\
\hline & 39 & Okumusashi & Sayamamidori $\times$ Yamatomidori & $\grave{4}$ & & 78 & Seishin oolong & (Derived from Taiwan) & \\
\hline & 40 & Okunoyama* & (Selection of Uji landrace) & & & 79 & Shizu-inzatsu 131 & (Selection of a natural cross population of Manipur no. 5) & \\
\hline
\end{tabular}

${ }^{a}$ Sample number is indicated in each cultivar for easy identification hereafter.

${ }^{b}$ Cultivars whose SSR allele data were fully obtained in this study are indicated with asterisks.

${ }^{c}$ Origins and parentages $(q \times \widehat{)})$ of cultivars are shown if described (full references are listed in Supplemental Text 1). Question marks mean unknown parentages. Parentages confirmed in this study are indicated with larger bold text, in which candidate parents newly predicted in this study are indicated with square brackets and filled stars $(\star)$ on the right. Parentages, in which both of the parents were confirmed, are indicated with open stars $(\xi)$ on the right. Cultivars directly selected from landraces or populations are shown in parentheses.

${ }^{d}$ Cultivars whose pedigrees have previously been suspected and corrected.

(Kubo et al. 2019). Allele data were treated as missing data (Supplemental Table 2) as three alleles were detected in triploid cultivars ('Hatsumidori' [12] and 'Makinoharawase' [24]; Table 1), and all allele data of these two cultivars were omitted from the estimation of genetic polymorphic parameters to avoid potential errors. The 32 SSR markers (MSG0083-MSE0335; Taniguchi et al. 2012) (Supplemental Table 5) were further used for genotyping 'Asahi' [3], 'Houshun' [16], 'Mie ryokuhou no. 1' [26], 'Rokurou' [42], 'Samidori' [46], 'Surugawase' [51], 'Tenmyo' [54], 'Yabukita' [61], 'Yamanoibuki' [65], and 'Yamatomidori' [66] using fluorescent-labeled primers or a post-labeling method (Shimizu and Yano 2011). Estimation of genetic polymorphic parameters and simulation of parentage analysis were conducted with Cervus 3.03 (Kalinowski et al. 2007) as reported previously (Kubo et al. 2009). LOD (natural log of the likelihood ratio) scores for tests, in which the most likely candidate parent is the true parent or not, were calculated with Cervus 3.03. In each of the 12 cultivars whose biparental pedigrees were newly identified or confirmed (Supplemental Table 4, Supplemental Fig. 1), only a single parent pair without mismatch in the SSR locus as well as the pedigree was identified, although more than one parent pair were predicted for some cultivars. In such cases, the identified parents mostly showed the highest LOD score (data not shown).

\section{Chloroplast DNA analysis for confirmation of maternity}

PCR amplification was performed for three chloroplast intergenic spacer regions (ndhF-rpl32, trnSGG-trnSr, and trnSf1-trnGGG; Wambulwa et al. 2016) in 23 cultivars (Supplemental Table 6). PCR products were directly sequenced after purification. The nucleotide sequences determined in this study are available in the DDBJ/EMBL/ GenBank databases under accession numbers LC630487LC630555. 


\section{Results}

Validation of SSR markers for the parentage analysis of three tea cultivars with known pedigrees

To assess the effectiveness of the DNA markers in the given samples, eight CAPS and nine SSR markers were compared to analyze the known pedigrees of 'Harumidori' [10], 'Minekaori' [29], and 'Saemidori' [44]. There was no incongruence in their pedigrees based on the eight CAPS markers, as the offspring always shared alleles with their maternal or paternal parent (Supplemental Table 1, upper panel, red, blue, and gray boxes), confirming their pedigrees. Similar results were obtained using the nine SSR markers (Supplemental Table 1, lower panel), but the average number of alleles was higher in the SSRs (3.6) than in the CAPSs (2.1). Therefore, we used the SSR markers to detect the alleles more accurately and examine the pedigrees more clearly.

\section{Polymorphisms of the SSR markers}

In the genotypes of 77 tea cultivars with the nine SSR markers (Supplemental Table 2) excluding two triploid cultivars, the mean number of alleles $\left(N_{\mathrm{A}}\right)$ was 14.22 per locus (Supplemental Table 3). This value was higher than that reported previously in 44 cultivars $(8.61$; Kubo et al. 2019) probably because of the increased number of cultivars examined in the present study. Mean values of the observed $\left(H_{\mathrm{O}}\right)$ and expected heterozygosities $\left(H_{\mathrm{E}}\right)$, and the polymorphic information content $(P I C)$ in this study were $0.7922,0.7910$, and 0.7624 , respectively; the $H_{\mathrm{O}}$ and $H_{\mathrm{E}}$ values were similar to but slightly lower than those in the previous study (Kubo et al. 2019). The null allele frequency estimate (F (Null)) of the marker MSG0699 was the highest (0.0282) among the nine SSR markers. There was no significant deviation from the Hardy-Weinberg equilibrium in any of the nine markers (data not shown).

\section{Parentage analysis of the tea cultivars}

Parentage analysis was performed for 79 cultivars based on the nine SSR markers. Biparental origins were identified or confirmed in nine cultivars, 'Houshun' [16], 'Mie ryokuhou no. 1' [26], 'Surugawase' [51], 'Tenmyo' [54], 'Yamanoibuki' [65], 'Koushun' [20], 'Okumusashi' [39], 'Sofu' [50], and 'Toyoka' [56] (Supplemental Table 4, Supplemental Fig. 1). Including the three cultivars, 'Harumidori' [10], 'Minekaori' [29], and 'Saemidori' [44], whose parentages were already confirmed (Supplemental Table 1), biparental origins were predicted for 12 cultivars (Table 1, filled and open stars). Notably, the candidate parents of 'Houshun' [16], 'Mie ryokuhou no. 1' [26], 'Surugawase' [51], 'Tenmyo' [54], and 'Yamanoibuki' [65] were newly identified in the present parentage analysis (Table 1, brackets and filled stars, Supplemental Table 4 and Supplemental Fig. 1, larger bold text). In the 12 parent-offspring relationships, all showed positive LOD scores and there was no SSR locus mismatch (Supplemental Table 4). For example, a 300-bp allele of the marker MSG0403 in 'Samidori' [46] was shared by 'Houshun' [16] and Tenmyo [54] (Table 2, red and gray boxes). 'Rokurou' [42] and 'Asahi' [3] were most likely the other parents of 'Houshun' [16] and 'Tenmyo' [54], respectively, as evidenced by the fact that there was no incongruence in the SSR markers between each of them (Table 2, blue and gray boxes). To confirm the five newly identified parentage results ('Houshun' [16], 'Mie ryokuhou no. 1' [26], 'Surugawase' [51], 'Tenmyo' [54], and 'Yamanoibuki' [65]), the number of examined SSR markers was increased to 41 in total. Again, this comparison showed no discrepancy in the five cultivars and their predicted parental cultivars (Supplemental Table 5, red, blue, and gray boxes). Uniparental origins were also confirmed for 25 cultivars (Supplemental Table 4, 'Asanoka' [4]-'Tsuyuhikari' [57]), but the remaining parent for each was unassigned.

\section{Confirmation of maternity for the determined parentages}

Chloroplast DNAs were analyzed to confirm the maternity of the present parentages. Three and one nucleotide polymorphisms were detected in the $n d h F-r p l 32$ and trnSf1-trnGGG loci, respectively (Supplemental Table 6). Seven cultivars ('Mie ryokuhou no. 1' [26], 'Harumidori' [10], 'Koushun' [20], 'Minekaori' [29], 'Saemidori' [44], 'Sofu' [50], and 'Toyoka' [56]) shared polymorphisms with their predicted maternal parents (Supplemental Table 6, red box), confirming their maternal parentages. Maternity was not confirmed for the other five cultivars, as there was no polymorphism in their tested chloroplast DNA.

\section{Discussion}

In this study, we performed parentage analyses of selected tea cultivars in Japan based on SSR markers, as to date, only a limited number of these analyses have previously been reported. The present study involved 79 cultivars, representing finer and larger scales of parentage analysis of tea cultivars in Japan than the previous reports. The 37 pedigrees were successfully confirmed or predicted from the 79 cultivars (Table 1, larger bold text). Notably, candidate parents ('Asahi' [3], 'Rokurou' [42], and 'Yamatomidori' [66]) were newly identified for the cultivars 'Houshun' [16], 'Mie ryokuhou no. 1' [26], 'Surugawase' [51], 'Tenmyo' [54], and 'Yamanoibuki' [65] (Table 1, brackets and filled stars, Supplemental Table 4 and Supplemental Fig. 1, larger bold text). 'Asahi' [3] is one of the best tencha cultivars, whereas 'Samidori' [46] is suitable for tencha and gyokuro; both of these cultivars were selected from Kyoto landraces by breeders in the private sector. Both were planted next to each other as they were expected to introduce their superior traits for tencha to their offspring ('Tenmyo' [54]) during its breeding process (our unpublished information).

The present study reveals that 'Rokurou' [42] is a 
Table 2. Genotypes of nine SSR markers in 'Tenmyo', 'Houshun', 'Surugawase', 'Yamanoibuki', 'Mie ryokuhou no. 1', and their predicted parental cultivars

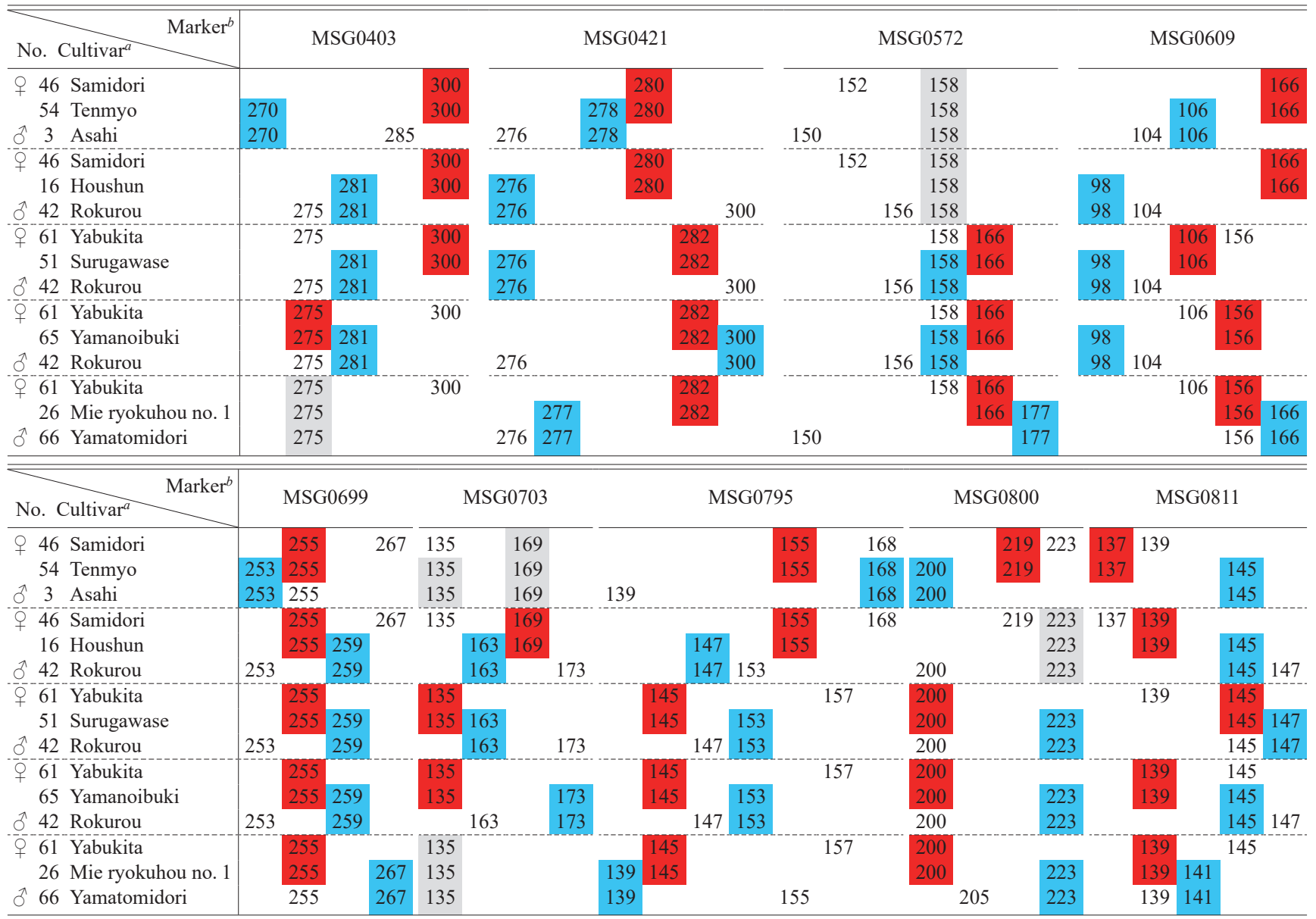

a The maternity and paternity ( $q$ and $\hat{\sigma}$, respectively) of cultivars were assigned based on the described pedigrees and the prediction in this study.

${ }^{b}$ Allele sizes (bp) are indicated below marker names. For each combination of three cultivars, alleles shared with the predicted maternal and paternal parents are colored in red and blue, respectively. Alleles of unclear parental origins are colored in gray. See online article for color version of this table.

candidate parent of 'Houshun' [16], 'Surugawase' [51], and 'Yamanoibuki' [65] (Table 1, Supplemental Table 4, Supplemental Fig. 1). 'Surugawase' [51] and 'Yamanoibuki' [65] are the offsprings of 'Yabukita' [61], bred by Shizuoka Prefecture (Kuranuki et al. 1997, Oishi and Hitaka 1966). 'Rokurou' [42] was selected from a landrace of unknown origin (Agriculture, Forestry and Fisheries Research Council 1968). Part of such pedigrees was in a good agreement with the inheritance of the disease resistance. 'Surugawase' [51] and 'Rokurou' [42] have strong resistance to gray blight disease, whereas 'Yabukita' [61] is susceptible to it (Takeda 2003). The genotypes of these cultivars were predicted to be $\mathrm{Pl}_{1} \mathrm{pl}_{1} p l_{2} \mathrm{pl}_{2}$ ('Surugawase' [51]), $\mathrm{Pl}_{1} \mathrm{pl}_{1} \mathrm{Pl}_{2} \mathrm{pl} \mathrm{l}_{2}$ ('Rokurou' [42]), and $\mathrm{pl}_{1} \mathrm{pl}_{1} \mathrm{pl}_{2} \mathrm{pl}_{2}$ ('Yabukita' [61]), where $\mathrm{Pl}_{1}$ and $\mathrm{Pl}_{2}$ confer strong and moderate levels of resistance, respectively (Supplemental Fig. 2; Takeda 2003). It is reasonable to infer that the haplotype $P l_{l} p l_{2}$, derived from 'Rokurou' [42], combined with $p l_{1} p l_{2}$ from 'Yabukita' [61] to generate the $P l_{1} p l_{1} p l_{2} p l_{2}$ genotype of
'Surugawase' [51] (Supplemental Fig. 2A). 'Houshun' [16] also has strong resistance to gray blight disease, whereas the resistance of its parent 'Samidori' [46] is slightly low (Kyoto Prefectural Tea Research Institute 2004a), suggesting that its strong resistance $\left(P l_{l}\right)$ is derived from 'Rokurou' [42] (Supplemental Fig. 2B). Hybridization with 'Rokurou' [42] could have provided the disease resistance trait in 'Houshun' [16] and 'Surugawase' [51]. 'Mie ryokuhou no. 1' [26] is the offspring of 'Yabukita' [61] (Ikeda et al. 1996). Its paternal parent was successfully predicted to be 'Yamatomidori' [66] in this study. 'Mie ryokuhou no. 1' [26] and 'Yamatomidori' [66] are late maturing cultivars, whereas 'Yabukita' [61] is a medium maturing one (Agriculture, Forestry and Fisheries Research Council 1968, Ikeda et al. 1996). The late maturing trait of 'Mie ryokuhou no. 1' [26] may be derived from 'Yamatomidori' [66].

The maternity for the pedigrees of the 12 cultivars was tested based on the maternally inherited chloroplast DNA. 
This approach was successful in seven of the 12 cultivars (Supplemental Table 6). The sequences were monomorphic in the other five cultivars despite the testing of several other chloroplast markers (data not shown). This was probably because of the lower mutation rate in the chloroplast DNA than in the nuclear DNA (Wolfe et al. 1987) as previously reported (e.g. Wambulwa et al. 2016). Irrespective of such limitations, we assumed that the probability of misidentifying maternal plants is low as mistakes in the crossbreeding of tea can occur more frequently during the process of pollination than seed harvesting, and therefore more frequently in paternal than maternal parents (Tanaka et al. 2001).

In conclusion, the present parentage analysis based on SSR markers has confirmed tens of the described pedigrees in the tea cultivars, and especially have unlabeled the parents of 'Houshun' [16], 'Mie ryokuhou no. 1' [26], 'Surugawase' [51], 'Tenmyo' [54], and 'Yamanoibuki' [65]. This parentage analysis improves our knowledge of tea pedigrees and will aid in the development of new cultivars with superior traits in breeding programs.

\section{Author Contribution Statement}

NK, TM, YM and MK designed this study. TM and YH maintained tea samples and helped in sampling. NK and CY performed the genotyping, genetic diversity and parentage analyses. NK, TM, YM and MK performed interpretation of results. NK drafted the manuscript. All authors read and approved the final manuscript.

\section{Acknowledgments}

We thank Mr. M. Hirano, Mr. H. Sawasaki, Mr. H. Katai, and Mr. Y. Kitao for valuable comments, Univ. Farm, Fac. Life Environ. Sci., Kyoto Pref. Univ., Inst. Fruit Tree Tea Sci., NARO, Shizuoka Pref. Res. Inst. Agric. Forest., Saitama Tea Res. Inst., Tea Ind. Res. Cent., and Yamato Tea Res. Cent., Nara Pref. Agric. Res. Dev. Cent. for providing tea leaf samples, and Ms. H. Kasaoka for technical help. This work was partly supported by the Academic Contribution to the Region (ACTR) grant from Kyoto Prefectural University and by Grant-in-Aid for Scientific Research (C) (20K12374) from Japan Society for the Promotion of Science to NK.

\section{Literature Cited}

Agriculture, Forestry and Fisheries Research Council (1968) Yabukita, Rokuro, Yamatomidori. New Tea Cultivar (Cha no Shinhinshu). Agriculture, Forestry and Fisheries Research Council, Tokyo, pp. 23-28, 37-42 (in Japanese).

Chen, Z.M. and L. Chen (2012) Delicious and healthy tea: an overview. In: Chen, L., Z. Apostolides and Z.M. Chen (eds.) Global Tea Breeding: Achievements, Challenges and Perspectives. Springer Business + Media, Berlin Heidelberg, pp. 1-11.

Ikeda, T., K. Ohtani, N. Tachibana and M. Yoshida (1996) A newly registered tea variety 'Mie ryokuhou no. 1'. Bull. Mie Agric. Tech. Cent. 24: 27-34 (in Japanese with English summary).

Kalinowski, S.T., M.L. Taper and T.C. Marshall (2007) Revising how the computer program CERVUS accommodates genotyping error increases success in paternity assignment. Mol. Ecol. 16: 10991106.

Katoh, M., M. Omori and Y. Katoh (2011) Identification of Japanese green tea cultivars using DNA fragment polymorphisms. J. Jpn. Soc. Food Sci. Technol. 58: 421-427 (in Japanese with English summary).

Kaundun, S.S. and S. Matsumoto (2003) Identification of processed Japanese green tea based on polymorphisms generated by STSRFLP analysis. J. Agric. Food Chem. 51: 1765-1770.

Kaundun, S.S. and S. Matsumoto (2004) PCR-based amplicon length polymorphisms (ALPs) at microsatellite loci and indels from noncoding DNA regions of cloned genes as a means of authenticating commercial Japanese green teas. J. Sci. Food Agric. 84: 895-902.

Kitajima-Ujihara, T. (2013) Studies on cultivar identification of green tea materials with DNA markers. Ph.D. Thesis, Kyoto University, Kyoto (in Japanese).

Kubo, N., M. Hirai, A. Kaneko, D. Tanaka and K. Kasumi (2009) Classification and diversity of sacred and American Nelumbo species: the genetic relationships of flowering lotus cultivars in Japan using SSR markers. Plant Genet. Resour. 7: 260-270.

Kubo, N., Y. Mimura, T. Matsuda, A.J. Nagano, N. Hirai, S. Higashimoto, H. Yoshida, N. Uemura and T. Fujii (2019) Classification of tea (Camellia sinensis) landraces and cultivars in Kyoto, Japan and other regions, based on simple sequence repeat markers and restriction site-associated DNA sequencing analysis. Genet. Resour. Crop Evol. 66: 441-451.

Kuranuki, Y., M. Aono-Shibata, T. Nagatani, Y. Nakamura and T. Hidaka (1997) Newly early budding tea cultivar, Yamanoibuki. Bull. Shizuoka Tea Exp. Stn. 21: 1-11 (in Japanese with English summary).

Kyoto Prefectural Tea Research Institute (2004a) A new candidate for gyokuro cultivar, "53-7" with superior aroma and the earliest budding (Komi ni sugureru gokuwase gyokuro-yo shinhinshu koho "53-7"). In: Western Agricultural Research Center, Research Highlights 2003 (Heisei 15 Nendo, Kinki Chugoku Shikoku Nogyokenkyu Seikajoho). Western Agricultural Research Center, National Agricultural Research Organization, Fukuchiyama, pp. 511-512 (in Japanese).

Kyoto Prefectural Tea Research Institute (2004b) A new candidate for tencha cultivar, "53-8" suitable for mechanical harvesting with a good quality and high yield (Kikai-zumi nimo tekisuru ryoshitsu tashu tencha-yo shinhinshu koho "53-8"). In: Western Agricultural Research Center, Research Highlights 2003 (Heisei 15 Nendo, Kinki Chugoku Shikoku Nogyokenkyu Seikajoho). Western Agricultural Research Center, National Agricultural Research Organization, Fukuchiyama, pp. 513-514 (in Japanese).

Liu, S., H. Liu, A. Wu, Y. Hou, Y. An and C. Wei (2017) Construction of fingerprinting for tea plant (Camellia sinensis) accessions using new genomic SSR markers. Mol. Breed. 37: 93.

Matsumoto, S., A. Takeuchi, M. Hayatsu and S. Kondo (1994) Molecular cloning of phenylalanine ammonia-lyase cDNA and classification of varieties and cultivars of tea plants (Camellia sinensis) using the tea PAL cDNA probe. Theor. Appl. Genet. 89: 671-675.

Matsumoto, S. (2006) Studies on differentiation of Japanese tea cultivars (Camellia sinensis) according to the genetic diversity of phenylalanine ammonia-lyase. Bull. Natl. Inst. Veg. Tea Sci. 5: 63-111 (in Japanese with English summary). 
Meegahakumbura, M.K., M.C. Wambulwa, K.K. Thapa, M.M. Li, M. Möller, J.C. Xu, J.B. Yang, B.Y. Liu, S. Ranjitkar, J. Liu et al. (2016) Indications for three independent domestication events for the tea plant (Camellia sinensis (L.) O. Kuntze) and new insights into the origin of tea germplasm in China and India revealed by nuclear microsatellites. PLoS ONE 11: e0155369.

Meegahakumbura, M.K., M.C. Wambulwa, M.M. Li, K.K. Thapa, Y.S. Sun, M. Möller, J.C. Xu, J.B. Yang, J. Liu, B.Y. Liu et al. (2018) Domestication origin and breeding history of the tea plant (Camellia sinensis) in China and India based on nuclear microsatellites and cpDNA sequence data. Front. Plant Sci. 8: 2270.

Merritt, B.J., T.M. Culley, A. Avanesyan, R. Stokes and J. Brzyski (2015) An empirical review: characteristics of plant microsatellite markers that confer higher levels of genetic variation. Appl. Plant Sci. 3: 1500025 .

Ni, S., M.Z. Yao, L. Chen, L.P. Zhao and X.C. Wang (2012) Germplasm and breeding research of tea plant based on DNA marker approaches. In: Chen, L., Z. Apostolides and Z.M. Chen (eds.) Global Tea Breeding: Achievements, Challenges and Perspectives. Springer Business + Media, Berlin Heidelberg, pp. 361376.

Ohsako, T., T. Ohgushi, H. Motosugi and K. Oka (2008) Microsatellite variability within and among local landrace populations of tea, Camellia sinensis (L.) O. Kuntze, in Kyoto, Japan. Genet. Resour. Crop Evol. 55: 1047-1053.

Oishi, S. and T. Hitaka (1966) On the early maturing variety "Surugawase" for green tea. Bull. Shizuoka Pref. Tea Exp. Stn. 1: 1-11 (in Japanese).

Shimizu, T. and K. Yano (2011) A post-labeling method for multiplexed and multicolored genotyping analysis of SSR, indel and SNP markers in single tube with bar-coded split tag (BStag). BMC Res. Notes 4: 161 .

Takeda, Y. and T. Toyao (1980) Identification and classification of the green tea varieties by the morphological characters of flower organs. Tea Res. J. 52: 1-6 (in Japanese with English summary).

Takeda, Y., K. Wada, A. Nesumi, N. Ikeda, S. Kondo, M. Hachinohe and Y. Yanase (1991) 'Saemidori', a newly registered early tea cultivar with high quality. Bull. Natl. Res. Inst. Veg. Ornam. Plants Tea Ser. B Tea 4: 1-15 (in Japanese with English summary).

Takeda, Y., A. Nesumi, K. Wada, T. Saba, H. Omae, J. Tanaka, S. Kondo, T. Takyu, N. Ikeda and M. Hachinohe (2002) A new cultivar, 'Harumidori', for green tea. Bull. Natl. Inst. Veg. Tea Sci. 1: 1-13 (in Japanese with English summary).

Takeda, Y. (2003) Phenotypes and genotypes related to tea gray blight disease resistance in the genetic resources of tea in Japan. Jpn. Agric. Res. Q. 37: 31-35.

Tamaki, I., T. Kuze, K. Hirota and M. Mizuno (2016) Genetic variation and population demography of the landrace population of Camellia sinensis in Kasuga, Gifu Prefecture, Japan. Genet. Resour. Crop Evol. 63: 823-831.

Tan, L.Q., M. Peng, L. Xu, L. Wang, S. Chen, Y. Zou, G.N. Qi and H. Cheng (2015) Fingerprinting 128 Chinese clonal tea cultivars using SSR markers provides new insights into their pedigree relationships. Tree Genet. Genomes 11: 90.
Tan, L.Q., Q.L. Liu, B. Zhou, C.J. Yang, X. Zou, Y.Y. Yu, Y. Wang, J.H. Hu, Y. Zou, S.X. Chen et al. (2019) Paternity analysis using SSR markers reveals that the anthocyanin-rich tea cultivar 'Ziyan' is self-compatible. Sci. Hortic. 245: 258-262.

Tanaka, J. and S. Yamaguchi (1996) Use of RAPD markers for the identification of parentage of tea cultivars. Bull. Natl. Res. Inst. Veg. Ornam. Plants Tea. Ser. B Tea 9: 31-36 (in Japanese with English summary).

Tanaka, J., N. Yamaguchi and Y. Nakamura (2001) Pollen parent of tea cultivar Sayamakaori with insect and cold resistance may not exist. Breed. Res. 3: 43-48 (in Japanese with English summary).

Tanaka, J. (2012) Japanese tea breeding history and the future perspective. In: Chen, L., Z. Apostolides and Z.M. Chen (eds.) Global Tea Breeding: Achievements, Challenges and Perspectives. Springer Business + Media, Berlin Heidelberg, pp. 227-239.

Taniguchi, F., K. Furukawa, S. Ota-Metoku, N. Yamaguchi, T. Ujihara, I. Kono, H. Fukuoka and J. Tanaka (2012) Construction of a high-density reference linkage map of tea (Camellia sinensis). Breed. Sci. 62: 263-273.

Taniguchi, F., K. Kimura, T. Saba, A. Ogino, S. Yamaguchi and J. Tanaka (2014) Worldwide core collections of tea (Camellia sinensis) based on SSR markers. Tree Genet. Genomes 10: 15551565.

Ueno, S. and T. Furuno (1989) A newly registered tea cultivar "Minekaori" suitable for pan-fried tea. Kyushu Agric. Res. 51: 59 (in Japanese).

Ujihara, T., R. Ohta, N. Hayashi, K. Kohata and J. Tanaka (2009) Identification of Japanese and Chinese green tea cultivars by using simple sequence repeat markers to encourage proper labeling. Biosci. Biotechnol. Biochem. 73: 15-20.

Ujihara, T., F. Taniguchi, J. Tanaka and N. Hayashi (2011) Development of expressed sequence tag (EST)-based cleaved amplified polymorphic sequence (CAPS) markers of tea plant and their application to cultivar identification. J. Agric. Food Chem. 59: $1557-1564$.

Wambulwa, M.C., M.K. Meegahakumbura, S. Kamunya, A. Muchugi, M. Möller, J. Liu, J.C. Xu, S. Ranjitkar, D.Z. Li and L.M. Gao (2016) Insights into the genetic relationships and breeding patterns of the African tea germplasm based on nSSR markers and cpDNA sequences. Front. Plant Sci. 7: 1244.

Wambulwa, M.C., M.K. Meegahakumbura, S. Kamunya, A. Muchugi, M. Möller, J. Liu, J.C. Xu, D.Z. Li and L.M. Gao (2017) Multiple origins and a narrow genepool characterise the African tea germplasm: concordant patterns revealed by nuclear and plastid DNA markers. Sci. Rep. 7: 4053.

Wolfe, K.H., W.H. Li and P.M. Sharp (1987) Rates of nucleotide substitution vary greatly among plant mitochondrial, chloroplast, and nuclear DNAs. Proc. Natl. Acad. Sci. USA 84: 9054-9058.

Xia, E.H., W. Tong, Q. Wu, S. Wei, J. Zhao, Z.Z. Zhang, C.L. Wei and X.C. Wan (2020) Tea plant genomics: achievements, challenges and perspectives. Hortic. Res. 7: 7.

Zhang, W., Y. Zhang, H. Qiu, Y. Guo, H. Wan, X. Zhang, F. Scossa, S. Alseekh, Q. Zhang, P. Wang et al. (2020) Genome assembly of wild tea tree DASZ reveals pedigree and selection history of tea varieties. Nat. Commun. 11: 3719. 